\title{
Hair cortisol in horses (Equus caballus) in relation to management regimes, personality and breed
}

Mathilde Sauveroche, Josefine Henriksson, Elvar Theodorsson, Ann-Charlotte B. Svensson Holm and Lina Roth

The self-archived postprint version of this journal article is available at Linköping University Institutional Repository (DiVA):

http://urn.kb.se/resolve?urn=urn:nbn:se:liu:diva-163937

N.B.: When citing this work, cite the original publication.

Sauveroche, M., Henriksson, J., Theodorsson, E., Svensson Holm, A. B., Roth, L., (2020), Hair cortisol in horses (Equus caballus) in relation to management regimes, personality and breed, Journal of Veterinary Behavior. https://doi.org/10.1016/j.jveb.2019.12.002

Original publication available at:

https://doi.org/10.1016/j.jveb.2019.12.002

Copyright: Elsevier

http://www.elsevier.com/

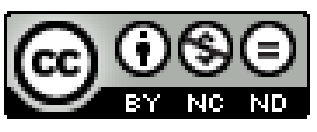


1 Hair cortisol in horses (Equus caballus) in relation to management regimes, 2 personality and breed

\section{Mathilde Sauveroche ${ }^{1}$, Josefine Henriksson ${ }^{1}$, Elvar Theodorsson ${ }^{2}$, Ann-Charlotte Svensson} Holm ${ }^{1}$, Lina S.V. Roth ${ }^{1 *}$

${ }^{1}$ IFM Biology, AVIAN Behavioural Genomics and Physiology group, Linköping University, 58183 Linköping, Sweden.

${ }^{2}$ Department of Clinical and Experimental Medicine, Linköping University, 58185 Linköping, Sweden.

*Corresponding author: lina.roth@liu.se

\begin{abstract}
Hair cortisol is a promising biomarker to measure long-term stress since cortisol is incorporated into the hair shaft as it grows. However, few studies have previously assessed hair cortisol concentrations (HCC) in horses. In this study, HCC was evaluated in both mane hair from the neck and body hair from the withers in 153 horses of different breeds, from seven different stables with three different management regimes (Free-roaming horses, Riding school horses, Trotter horses). In addition, 4 hours of behavioural observations were performed at each stable, and for 43 of the horses, a personality survey was completed. Mane and withers HCC correlated moderately, but significantly $\left(r_{s}=0.48, p<0.001\right)$. Differences between the stables were found for both mane and withers hair (both $p<0.01$ ) and the stable with lowest HCC also showed highest occurrences of positive social and resting behaviours (both $\mathrm{p}<0.01$ ). There were no significant differences in $\mathrm{HCC}$ between the management regimes even though Free-roaming horses showed less negative social behaviours compared to Riding school horses $(p=0.041)$ and Trotter horses $(p=0.055)$. The personality traits Dominance, Anxiousness, and Excitability revealed weak to moderate correlations with mane HCC $\left(r_{s}=-0.34, p=0.027 ; r_{s}=-0.46, p=0.002 ; r_{s}=-0.31, p=0.043\right.$ respectively) which might suggest that personality could also be related to long-term stress levels in horses.
\end{abstract}

Keyword: Horse, Cortisol, Behaviour, Stress, Welfare, Management, Personality 


\section{Introduction}

The use of horses has varied since the time of domestication, from being bred for food to being used as a mean of transport, and finally, for sports and leisure purposes (Clutton-Brock 1981; Thorell \& Hedenborg 2015). Despite being one of the most popular farm animals, little is known about how different management regimes affect the health and welfare of horses. Welfare in horses is closely connected to management and housing practices since it refers to the individual's state and coping responses in relation to its environment (Broom 1991). If an animal is confronted with an inadequate environment it will be likely to experience stress, which can be reflected in its behaviour (Cooper \& Mason 1998; Visser et al. 2012). The behaviour of horses is being thoroughly studied to better fulfil their needs (Costa et al. 2014; Viksten et al. 2017). However, welfare includes both physical and mental aspects, thus its assessment needs to consider both physiological and behavioural parameters (Blokhuis et al. 2010).

Stress strongly affects animal welfare (Keeling \& Jensen 2009) and can be defined as an unpredictable, uncontrollable environmental challenge that can result in adverse consequences for an animal, eventually reducing fitness (Broom 1991; Koolhaas et al. 2011). The immediate physiological response to stress is increased activity in the sympathetic nervous system, causing increased heart rate (Gácsi et al. 2013), as well as an increased activity of the hypothalamic-pituitary-adrenal axis (Koolhaas et al. 2011) (HPA). Increased heart rate and elevated cortisol concentrations in blood and saliva are therefore strongly associated with acute stress (Koolhaas et al. 2011) and it is possible to measure and monitor them even if susceptible to sampling stress. Although short-term stress can redirect energy to respond to a potential dangerous situation and hence does not necessarily imply compromised welfare, if prolonged, stress can negatively affect an animal's health (Chrousos 2000). One promising method to assess long-term stress in animals is to measure concentrations of cortisol incorporated in hair (Davenport et al. 2006; Russell et al. 2012; Roth et al. 2016). As the hair grows, cortisol from the blood is gradually incorporated, in effect forming a retrospective calendar of cortisol concentrations (Russell et al. 2012).

Although hair cortisol concentrations (HCC) have been assessed in several species (Davenport et al. 2006; Russell et al. 2012; Roth et al. 2016), only few recent studies have analysed HCC in horses. HCC in foals are shown to decrease after birth (Comin et al. 2012) and environmental factors such as temperature, rainfall and day length is suggested to have no influence on HCC (Montillo et al. 2014). A recent study carried out cortisol measures on both mane and tail hair in adult horses to assess long-term stress in these animals (Duran et al. 2017). Their results suggested that horse HCC can vary in response to exposure to a specific stressor. Hence, hair seems to be a promising matrix to measure long-term cortisol variation in horses.

The objectives of this project were to 1) determine if mane hair and body hair differed for measuring HCC in horses, 2) investigate whether different stables or management regimes have different impact on the stress status of the horses, using both HCC and behavioural analyses, and lastly, 3) HCC were correlated to personality traits in order to reveal whether different traits, that per definition should be consistent over time, could be related to HCC.

\section{Method}




\section{Subjects}

A total of 153 horses and ponies from seven different stables (A-G) in the southeast of Sweden (within $50 \mathrm{~km}$ ) were recruited, through personal contact, to participate in this study (Table 1). The age of the horses ranged from 3 to 28 years, but the age of two horses from stable E was unknown. Stable A and Stable B consisted of only Icelandic horses; Stable C contained both Icelandic horses and Shetland ponies; Stables D and E were both riding schools with horses and ponies of various breeds; Stable F and G contained Standardbred trotter horses. To simplify in the following text and figures, the stables will hereafter be referred to as Icelandic A, Icelandic B, Icelandic C, Riding school D, Riding school E, Trotter F and Trotter $\mathrm{G}$.

All horses included in the study were privately owned, their owners were informed about the study and gave their voluntary consent to participate. This study complies with the Swedish and European regulation for the use of animal subjects for research purposes. Since we only performed behavioural observations and obtained hair samples non-invasively from privately owned horses, ethical permission was not needed.

\section{Horse and management information}

The management style was determined during visits at the stables and from personal communication with owners and staff. Icelandic $A$ kept their horses outdoors during the day and indoors at night in large group boxes, Icelandic B and C kept their horses and Shetland ponies in large groups outdoors both day and night, Riding school D and E kept their horses and ponies in large groups outdoors during the day and in individual boxes at night which also was the case for the Trotter stables $F$ and $G$ even though some trotters were divided into smaller groups. Due to the different management regimes we grouped Icelandic $B$ and $C$ into a Free-roaming group, Riding school $D$ and $E$ into a Riding school group and Trotter racing $F$ and $G$ into a Trotter racing group (Table 1 ). Hence, Icelandic $A$ with a dissimilar housing than all other stables was not included when we tested differences for management regimes.

The staff of each stable was asked to answer questions about: age, sex, breed and estimated hierarchy rank of each horse (rank score was between 1-7 where 1 meant low rank and 7 meant high rank), but also how many hours the horses were outdoors on a regular day, how many hours they were exercised and if any horse was competing in any equestrian sport. All collected information about the horses participating in this study can be found in Supplement 1.

\section{Horse personality questionnaire}

Finally, staff was asked to complete a Horse personality questionnaire developed by Lloyd et al. (2007). The survey was translated into Swedish and contained 25 fully described adjectives (see Lloyd et al. 2007 for details) which the staff was asked to score for each horse using a seven-point scale: 1 "no expression", 4 "average" and 7 "total expression". The adjective scores for each horse were thereafter multiplied with the original Principal component analysis (PCA) loadings in order to achieve values on six different personality traits for each horse/pony (Lloyd et al. 2007): Dominance (high negative loadings for reliability, subordinate and equable, and high positive loadings for irritable, aggressive, stubborn, effective and eccentric), Anxiousness (high positive loadings for fearful, 
apprehensive, tense, insecure and suspicious), Excitability (high negative loading for slow and high positive loading for active, intelligent and excitable), Protection (high loadings for protective, motherly and understanding), Sociability (high loadings for popular, playful and sociable) and Inquisitiveness (high loadings for opportunistic and curious).

\section{Behavioural observations}

All observational sessions were performed between September and October 2017. Observations were carried out for two days per stable, each day separated from the other by at least two weeks and each day consisted of $4 \times 30$ min sessions. Hence, a total of eight sessions and four hours of observations were held at each stable between 8:30am and 12:15pm. Rotational focal sampling was used, switching between individuals in a randomised manner every two minutes. Both continuous sampling and 1/0 interval sampling with 30 second intervals was carried out (Table 2; Supplement 2). Social, stereotypical, locomotion and feeding-related behaviours were recorded at group level by completing ethogram worksheets at the time of the observations. Occurrence was noted for states and frequency for events. The same observer collected all behavioural data.

\section{Hair sampling and cortisol analyses}

During September 2017 two hair samples were obtained from 153 horses participating in this study. One of the two samples contained a strand of mane hair behind the head collar position which was cut using scissors. The second sample was cut using a clipper on the left side of the horse, in front of the withers and above the scapula, two fingers $(3-4 \mathrm{~cm})$ below the mane (Fig.1). Both samples were cut as close to the skin as possible and stored in plastic zip bags at room temperature until further analysis. The hair was always placed in the same direction in the plastic bag to be able to later analyse the correct part of the hair shaft.

One centimetre of the hair shafts closest to the skin was cut into smaller (1-2 $\mathrm{mm}$ ) pieces and weighed (approximately $7 \mathrm{mg}$-range: 6-8.5 mg-, the exact weight was recorded for further calculations). Cortisol extraction and analysis were done according to methods previously described (Karlén et al. 2011; Roth et al. 2016). In short, the hair samples were frozen in liquid nitrogen and pulverized with steel beads using a Tissue lyser II ( 2 min at 23 $\mathrm{Hz}$ ). Methanol $(1 \mathrm{ml})$ was added to each sample after which they had been shaken overnight in a tube shaker. On the following day the samples were centrifuged $\left(23 \mathrm{G}, 1 \mathrm{~min}, 4^{\circ} \mathrm{C}\right)$ and $800 \mu \mathrm{l}$ of the supernatant was taken and put in a Savant Speed-Vac Plus SC210A to evaporate $(1.5 \mathrm{~h})$. The remaining pellets were stored in the refrigerator $\left(4-8^{\circ} \mathrm{C}\right)$ until further analysis.

Finally, the pellets were dissolved in $150 \mu \mathrm{L}$ RIA buffer ( $\mathrm{pH}$ 7.4). Then $50 \mu \mathrm{L}$ of this dissolved solution was then taken and $100 \mu \mathrm{L}$ of primary antibody (Anti-cortisol rabbit antibody) was added giving a $30-40 \%$ binding to the radioligand. After a $48 \mathrm{~h}$ incubation period, $100 \mu \mathrm{L}$ of radioactive $\left({ }^{125} \mathrm{I}\right)$ conjugated cortisol was added to each tube, these were then incubated for $24 \mathrm{~h}$. After this incubation $75 \mu \mathrm{L}$ of Anti-Rabbit IgG SAC-CEL (Solid phase second anti-rabbit antibody coated cellulose suspension from Immunodiagnosticsystems, IDS $($ ) was added to all samples. After $30 \mathrm{~min}$, the reaction was stopped by adding $2 \mathrm{~mL}$ of water. The samples were then centrifuged for $15 \mathrm{~min}\left(3000 \mathrm{rpm} ; 4^{\circ} \mathrm{C}\right)$. The water was removed using a decanting tool and the samples were analysed using a PerkinElmer 2470 Wizard gamma counter which gave measures in CPM and $\mathrm{nmol} / \mathrm{L}$, later converted into pg of cortisol per $\mathrm{mg}$ of hair 
normalised by the initial weight of the cut hair. Samples were run in duplicates for validity and the inter-assay coefficient of variability was found to be $11.8 \%$, while the intra-assay coefficient of variability was $13.5 \%$.

\section{Data analysis}

HCC were not normally distributed (Shapiro-Wilk test $\mathrm{p}<0.05$ ) and therefore non-parametric tests were used. Spearman correlations (two-tailed) were used for all correlations, KruskalWallis tests were used to test differences between management regimes and breeds and when differences were found, follow-up pairwise comparisons with $p$-values adjusted by the Bonferroni correction for multiple tests were conducted. Sex differences were tested with Mann-Whitney $U$ test and the different hair types were tested with Wilcoxon Signed-Rank test.

Inter-observer reliability was accounted for by comparing data collected with a second observer in the first four hours of observation (Mann Whitney $U$ tests $p>0.05$ for all observed behaviours). All statistical tests were performed with SPSS statistic software (IBM, version 24).

\section{Results}

\section{Hair cortisol}

Hair samples were obtained from both mane and withers from 153 horses except for one horse in Riding school D where only the mane sample was obtained due to technical difficulties.

The HCC $(\mathrm{pg} / \mathrm{mg}$ ) measured in the mane (Median=6.0, SD=6.4, N=152) correlated moderately with the concentration in the withers body hair (Median=6.1, SD=8.6, N=151) $\left(\mathrm{N}=152, \mathrm{r}_{\mathrm{s}}=0.48, \mathrm{p}<0.001\right)$. This was also true when one outlier with withers $\mathrm{HCC}$ of 127 $\mathrm{pg} / \mathrm{mg}$ was removed $\left(\mathrm{N}=151, \mathrm{r}_{\mathrm{s}}=0.48, \mathrm{p}<0.001\right)$.

HCC between the stables differed significantly for both mane and withers hair (Fig. $2 \mathrm{~A} ; \chi^{2}(6$, $\mathrm{N}=152)=17,6, \mathrm{p}=0.007$ and $\chi^{2}(6, \mathrm{~N}=151)=22,8, \mathrm{p}=0.001$, respectively). In pairwise comparisons the mane $\mathrm{HCC}$ for Icelandic $\mathrm{A}$ (Median=4.2, SD=5.3, N=16) were significantly lower than that from Icelandic $B$ (Median=7.6, $S D=5.7, N=14, p=0.005$ ) and $C$ (Median=6.3, $\mathrm{SD}=2.1, \mathrm{~N}=25, \mathrm{p}=0.029$ ) and the withers $\mathrm{HCC}$ in Icelandic $\mathrm{A}$ (Median=4.2, $\mathrm{SD}=2.0, \mathrm{~N}=16$ ) were also significantly lower than that from Riding school $D$ (Median=10.1, $S D=21.1, N=44$, $\mathrm{p}=0.001$ ) and Trotter $\mathrm{F}$ (Median=7.0, SD=7.5, N=16, $\mathrm{p}=0.032$ ).

When comparing $\mathrm{HCC}$ for the different management regimes, that is Free-roaming horses (Icelandic B and C), Riding school horses (Riding school D and E) and Trotter racing horses (Trotter $F$ and $G$ ), there were no significant differences (mane: $\chi^{2}(2, N=84)=5.2, p=0.074$ and withers: $\left.\chi^{2}(2, N=135)=3.5, p=0.17\right)$.

HCC were compared for the largest breed groups (43 Icelandic horses, 12 Shetland ponies and 29 Standardbred trotters) and significant differences were found for the withers hair cortisol (Fig. 2B; withers: $\left.\chi^{2}(2, \mathrm{~N}=84)=9.1, \mathrm{p}=0.011\right)$. The Standardbred trotter horses (Median=7.0, SD=6.4, N=29) showed higher HCC than the Shetland ponies (Median=4.9, 
$\mathrm{SD}=1.9, \mathrm{~N}=12, \mathrm{p}=0.022$ ) and that was also a trend for the Icelandic horses (Median= 5.8, $\mathrm{SD}=4.2, \mathrm{~N}=43, \mathrm{p}=0.053)$. No significant results were found for the mane $\mathrm{HCC}\left(\chi^{2}(2\right.$,

Age did not correlate with HCC (mane: $N=150, r_{s}=0.07, p=0.39$; withers: $N=149, r_{s}=-0.09$, $p=0.27$ ), nor did sex affect the cortisol concentrations (mane: $N=152, U=2889, p=0.83$; withers: $\mathrm{N}=151, \mathrm{U}=2653, \mathrm{p}=0.55$ ). However, comparing the age within the different management regimes revealed that the Trotter racing horses were younger (Median= 5, SD= 2.9, $\left.\mathrm{N}=29 ; \chi^{2}(2, \mathrm{~N}=150)=49.5, \mathrm{p}=0.001\right)$ than both the Free-roaming horses (Median=14, $\mathrm{SD}=6.1, \mathrm{~N}=39, \mathrm{p}=0.001$ ) and Riding school horses (Median=12.0, $\mathrm{SD}=4.7, \mathrm{~N}=67, \mathrm{p}=0.001$ ).

Since the full survey (including a Horse personality questionnaire) was too time consuming for some stables, information provided for some of the horses, such as training hours was averaged (see Supplementary 1). No correlation between HCC and training hours per week was found. Moreover, the fact that the horses were competing in any equestrian sport did not significantly affect the $\mathrm{HCC}$ (mane: $\mathrm{N}=102, \mathrm{U}=703, \mathrm{p}=0.86$; withers: $\mathrm{N}=101, \mathrm{U}=713$, $p=0.99)$.

We received complete personality scores for 43 horses (however, one horse turned out to be an outlier in HCC and was not included in the personality correlations). Moderate to weak negative correlations were found between mane $\mathrm{HCC}$ and the following personality traits obtained from the Horse personality questionnaire (from stable $B, C$ and $D$ only): Dominance $\left(\mathrm{N}=42, \mathrm{r}_{\mathrm{s}}=-0.34, \mathrm{p}=0.027\right)$, Anxiousness $\left(\mathrm{N}=42, \mathrm{r}_{\mathrm{s}}=-0.46, \mathrm{p}=0.002\right)$ and Excitability $\left(\mathrm{N}=42, \mathrm{r}_{\mathrm{s}}=-\right.$ $0.31, p=0.043)$. Noteworthy, all three personality traits correlated positively with each other $(p<0.001)$ and no significant correlations were found between these traits and withers HCC or between personality traits and age of the horse. Also, reported hierarchy rank of the horse did not correlate with the HCC ( $p>0.05)$.

\section{Behaviour}

We found differences between stables for all behavioural categories (Table 1; Fig. 3A) except Negative social behaviours. Positive social behaviours $\left(\chi^{2}(6, N=56)=21.2, p=0.002\right)$ were most abundant in Icelandic $A$ and pairwise comparisons showed that Icelandic $A$ had significantly higher amount of positive social behaviours than both Icelandic B and Riding school $E$ (both $p<0.05)$. Resting behaviours differed significantly $\left(\chi^{2}(6, N=56)=26.8, p<0.001\right)$ where Icelandic $A(p<0.01)$, Icelandic $C(p<0.05)$ and Riding school $D(p<0.01)$ showed higher resting behaviour occurrences than Riding school E. Locomotion behaviours $\left(\chi^{2}(6, N=56)=15.9\right.$, $\mathrm{p}=0.014)$ were most frequent in Trotter $\mathrm{F}$ compared to both Icelandic $\mathrm{B}$ and Icelandic $\mathrm{C}$ $(p<0.05)$ and lastly, Feeding behaviours $\left(\chi^{2}(6, N=56)=23.0, p=0.001\right)$ were most abundant in Riding school E compared to Icelandic A, Icelandic B and Riding school $D(p<0.05)$.

Comparing behaviours for the three management regimes (Free-roaming horses, Riding Schools, Trotter racing) showed that negative social behaviours differed significantly (Fig. 3B; $\left.\chi^{2}(2, N=48)=7.8, p=0.020\right)$ and pairwise comparisons revealed Free-roaming horses to have lower negative social behaviour occurrences than Riding School horses $(p=0.041)$ and that these occurrences also tended to be lower compared to the Trotter racing horses $(p=0.055)$. Locomotion behaviours differed significantly $\left(\chi^{2}(2, N=48)=11.7, p=0.003\right)$ where Trotter racing horses showed higher occurrences than Free-roaming horses $(p=0.002)$. 


\section{Discussion}

In this study HCC from two different hair types/body locations in 153 horses were assessed. Cortisol concentrations in mane hair from the neck and body hair from the withers were positively correlated which suggests that both could be suitable for analysing HCC. Differences were found when comparing the seven stables regarding both HCC and behaviours. However, the management regimes and the age of the horses did not reveal any associations to the HCC.

In general, the HCC of the horses in this study (Fig. 2A,B) were lower or similar to previous reported results for hair cortisol concentrations in horses (Comin et al. 2012; Montillo et al. 2014; Duran et al. 2017). Moreover, during the behavioural observations, no stereotypical behaviours were recorded, which could indicate that the horses in this study were not subjected to high concentrations of long-term stress.

When comparing stables, the horses in Icelandic A stable revealed lowest HCC compared to the other stables included in this study. In addition, Icelandic A showed most positive social behaviours but also most resting behaviours during the outdoor group observations. It has previously been reported that horses having social interactions have lower faecal glucocorticoid concentrations than socially isolated horses (Yarnell et al. 2015). This supports our results, but requires further investigation in order to assess the causations rather than associations between behaviour and HCC.

There are most certainly many aspects influencing $\mathrm{HCC}$ and in this study we can only suggest associations between groups of horses and HCC. Since weather and temperature has earlier been suggested to have no effect on HCC in horses (Montillo et al. 2014) and since the horses in this study all lived in the south east of Sweden, weather aspects should not have influenced our HCC results. However, interestingly dogs have recently been shown to synchronize in HCC with their owner (Sundman et al. 2019), and whether they have access to garden or not, does not seem to influence the dog's HCC. Hence, it would have been interesting to measure the $\mathrm{HCC}$ of the stable staff, especially since we found differences in $\mathrm{HCC}$ between stables but not between management regimes. Still, the everyday lives of dogs and horses are rather different and speculations on causation for differences in horses' HCC cannot be resolved within the scope of this study.

Again, when comparing the management regimes, (Free-roaming horses, Riding Schools, Trotter stables), no differences were found in $\mathrm{HCC}$, however, the Free-roaming horses (Icelandic B and Icelandic C) showed lowest occurrences of negative social behaviours. The Free-roaming horses also revealed less locomotion behaviours compared to the Trotter racing horses. This could reflect the fact that since the Free-roaming horses were continuously outdoors, their activity bouts were likely to be spread out over the day, while the horses that were outdoors for a limited amount of time (e.g. the trotters) might have exercised more during the observations. The Trotter racing horses were also significantly younger than the horses in the other regimes in this study and contained only horses (Standardbred trotters) and no ponies which could have influenced their activity pattern (Piccione et al. 2011). Although exercise has been shown to increase cortisol concentrations 
(Gordon et al. 2007), we did not find increased HCCs in horses that showed more activity during the behavioural observations. Similarly, the herein reported training frequency did not seem to influence the measured HCC. However, it should be noted the observation sessions were relatively few and the observed differences in behaviours among stables and management regimes could have been influenced by numerous factors such as the size of the enclosure, the number of horses in the group, whether there was food available at the time of observations, etc. (Hogan et al. 1988; Yarnell et al. 2015; Hartmann et al. 2012), making it difficult to draw major conclusions of these results. Feeding behaviours also differed between the stables, but again, due to different management routines in which some horses were fed extra roughage when let out from the stable, this result should be interpreted with caution.

Interestingly, the HCC correlated negatively with the horses' and ponies' personality traits Dominance, Anxiousness and Excitability, but notably all those traits correlated with each other, and the correlations were weak, which might reveal difficulties of measuring personality with only a survey. Still, the lack of strong associations between personality traits and $\mathrm{HCC}$ is in line with a recent study where the dog's own personality had little effect on its HCC (Sundman et al. 2019). However, dominant individuals in primates have been shown to have lower cortisol concentrations than subordinates (Feng et al. 2016), but if the hierarchy is unstable it might instead increase stress levels in high-ranking individuals (Muller \& Wrangham 2004). In horses, faecal cortisol metabolite concentrations correlate negatively with hierarchy rank (Christensen et al. 2012). Hence, a horse that in the study was-through observation-ranked as high in the social hierarchy had low cortisol concentrations. This is in accordance with our results and since the horses in our stables were kept in relatively consistent groups, they had probably acquired a fixed hierarchy which contributed to the moderate negative correlation found between the dominance trait and HCC. The dominance scores in our study were obtained through a personality questionnaire with 25 welldescribed adjectives (Lloyd et al. 2007). The additional hierarchy rank score obtained initially from the staff and not accompanied with a full description did not correlate with neither cortisol nor the dominance trait. Hence, besides taking into account the hierarchy stability of participant animals, the difficulty to objectively assess personality traits in animals also needs to be considered when investigating the physiological relationship between hierarchy rank and stress. When it comes to the personality trait Anxiousness there are suggestions that chronic anxiety might downregulate the activity of the HPA-axis and hence lower the HCC (Steudte et al. 2011) which is consistent with our results. However, since the personality trait Excitability is closely connected to the activity of the horse, this moderate correlation is more difficult to explain and due to the absence of individual behavioural observations to validate the personality traits we will not speculate further on this.

As all the current behavioural results reflect behaviour at group level, it would be interesting for future studies to investigate the link between individual behaviour and HCC. Recent research on enclosure size has also found that the bigger the pasture, the fewer social interactions between horses take place (Majecka \& Klawe 2018), which might have had an effect on the social behaviour occurrences in this study. However, enclosure sizes for the different stables were not obtained in this study, and hence it would be interesting to also investigate pasture size in relation to both social behaviours, activity and HCC in future studies. 
When the largest breed groups in this study $(N>10)$ were analysed for possible hair cortisol differences (Icelandic horses from Icelandic A, B and C; Shetland ponies from Icelandic C and Standardbred trotters from Trotter F and G), the Standardbred trotters showed the highest HCC. However, as mentioned earlier, different management regimes and enclosure sizes could have affected these results. It has been previously reported that HCC do not differ between two tested breeds (Duran et al. 2017). For future breed comparisons, horses with similar management regimes should be studied and complemented with behavioural observations on an individual level.

Since a recent study (Duran et al. 2017) reported a difference between mane and tail HCC, a standardisation of sampling method and body location would be preferable for future comparative studies. We would suggest sampling the mane since it varied little in thickness, and length between horses compared to body hair. In addition, the mane hair did not require the use of a clipper.

In conclusion, the HCC from the two types of horse hair correlated significantly suggesting that this is a promising tool to assess long-term stress in horses. Interestingly, cortisol and behavioural differences were found between participating stables, where more positive social behaviours were associated with low HCC. Also, less negative social behaviours were observed in Free-roaming horses. For future studies we suggest observations of the behaviour on an individual basis to improve the comparisons with the individual HCC.

\section{Acknowledgement}

We are very grateful to all the stables who voluntarily welcomed us for this study, as well as all the stable staff involved. We thank Per Jensen, Ann-Sofie Sundman and Enya Van Poucke for discussions and appreciated comments on an earlier version of the manuscript and Åshild Olsen Faresjö for valuable lab advise.

\section{Author Statement}

The idea for the paper was conceived by LSVR and MS. The experiments were designed by LSVR and MS with help of JH. The behavioural observations were performed by MS with help of $\mathrm{JH}$ and lastly ET, ASH and MS were responsible for the analyzation in the lab. All authors reviewed the manuscript.

\section{Competing interests}

The authors declare no competing interests.

\section{Data Availability}

Behavioural data, individual information, management regimes and the hair cortisol concentrations of the horses are available in Supplementary 1 and Supplementary 2.

\section{Ethical treatment of animals}

All horses included in the study were privately owned, their owners were informed about the study and gave their voluntary consent to participate. This study complies with the Swedish and European regulation for the use of animal subjects for research purposes. Since 
we only performed behavioural observations and obtained hair samples non-invasively from privately owned horses ethical permission was not needed.

\section{References}

Blokhuis, H. J., Veissier, I., Miele, M., Jones, B., 2010. The Welfare Quality ${ }^{\circledR}$ project and beyond: Safeguarding farm animal well-being. ACTA AGR. SCAND. A-AN. 60, 129-140.

Broom, D.M., 1991. Animal welfare: concepts and measurement. J. Animal Sci. 69, 41674175.

Christensen, J.W., Ahrendt, L.P., Lintrup, R., Gaillard, C., Palme, R., Malmkvist, J., 2012. Does learning performance in horses relate to fearfulness, baseline stress hormone, and social rank? Appl. Anim. Behav. Sci. 140, 44-52.

Chrousos, G.P., 2000. Stress, chronic inflammation, and emotional and physical well-being: Concurrent effects and chronic sequelae. J. Allergy Clin. Immunol. 106, S275-S291.

Clutton-Brock, J., 1981. Domesticated animals from early times, British Museum (Natural History) and William Heinemann Ltd.

Comin, A., Veronesi, M.C., Montillo, M., Faustini, M., Valentini, S., Cairoli, F., Prandi, A., 2012. Hair cortisol level as a retrospective marker of hypothalamic-pituitary-adrenal axis activity in horse foals. Vet. J. 194, 131-132.

Cooper, J.J. \& Mason, G.J., 1998. The identification of abnormal behaviour and behavioural problems in stabled horses and their relationship to horse welfare: a comparative review. Equine Vet. J. 27, 5-9.

Costa, E.D., Murray, L., Dai, F., Canali, E., Minero, M., 2014. Equine on-farm welfare assessment: a review of animal-based indicators. Anim. Welf., 23, 323-341.

Davenport, M., Tiefenbacher, S., Lutz, C.K., Novak, M.A., Meyer, J.S., 2006. Analysis of endogenous cortisol concentrations in the hair of rhesus macaques. Gen. Comp. Endocrinol. 147, 255-261.

Duran, M.C., Janz, D.M., Waldner, C.L., Campbell, J.R., Marques, F.J., 2017. Hair Cortisol Concentration as a Stress Biomarker in Horses: Associations With Body Location and Surgical Castration. J. Equine Vet. Sci. 55, 27-33.

Feng, X., Wu, X., Morrill, R.J., Li, Z., Li, C., Yang, S., Li, Z., Cui, D., Lv, L., Hu, Z., Zhang, B., Yin, Y., Guo, L., Qin, D., Hu, X., 2016. Social correlates of the dominance rank and long-term cortisol levels in adolescent and adult male rhesus macaques (Macaca mulatta). Sci. Rep. 6:25431, 1-9.

Gácsi, M., Maros, K., Sernkvist, S., Faragó, T., Miklósi, Á., 2013. Human Analogue Safe Haven Effect of the Owner: Behavioural and Heart Rate Response to Stressful Social Stimuli in Dogs. PLoS ONE, 8, e58475-9.

Gordon, M.E., McKeever, K.H., Betros, C.L., Manso Filho, H.C., 2007. Exercise-induced alterations in plasma concentrations of ghrelin, adiponectin, leptin, glucose, insulin, and cortisol in horses. Vet. J. 173, 532-540.

Hartmann, E., Søndergaard, E., Keeling, L.J., 2012. Keeping horses in groups: A review. Appl. Anim. Behav. Sci. 136, 77-87.

Hogan, E.S., Houpt, K.A., Sweeney, K., 1988. The effect of enclosure size on social interactions and daily activity patterns of the captive Asiatic wild horse (Equus przewalskii). Appl. Anim. Behav. Sci. 21, 147-168.

Karlén, J., Ludvigsson, J., Frostell, A., Theodorsson, E., Faresjö, T., 2011. Cortisol in hair measured in young adults - a biomarker of major life stressors? BMC Clin. Pathol. 11, 1-6. 
460

461

462

463

464

465

466

467

468

469

470

471

472

473

474

475

476

477

478

479

480

481

482

483

484

485

486

487

488

489

490

491

492

493

494

495

496

497

498

499

500

501

502

503
Keeling, L., Jensen, P., 2009. Abnormal behavior, stress and welfare in The ethology of domestic animals. CABI Publishing.

Koolhaas, J.M., Bartolomucci, A., Buwalda, B., de Boer, S.F., Flügge, G., Korte, S.M., Meerlo, P., Murison, R., Olivier, B., Palanza, P., Richer-Levin, G., Sgoifo, A., Steimer, T., Stiedl, O., van Dijk, G., Wöhr, M., Fuchs, E., 2011. Stress revisited: a critical evaluation of the stress concept. Neurosci. Biobehav. Rev. 35, 1291-1301.

Lloyd, A. S., Martin, J. E., Bornett-Gauci, H. L. I., Wilkinson, R. G., 2007. Evaluation of a novel method of horse personality assessment: Rater-agreement and links to behaviour. Appl. Anim. Behav. Sci. 105, 205-222.

Majecka, K., Klawe, A., 2018. Influence of Paddock Size on Social Relationships in Domestic Horses. J. Appl. Anim. Welf. Sci. 21, 8-16.

Montillo, M., Comin, A., Corazzin, M., Peric, T., Faustini, M., Veronesi, M.C., Valentini, S., Bustaffa, M., Prandi, A., 2014. The Effect of Temperature, Rainfall, and Light Conditions on Hair Cortisol Concentrations in Newborn Foals. J. Equine Vet. Sci. 34, 774-778.

Muller, M.N., Wrangham, R.W., 2004. Dominance, cortisol and stress in wild chimpanzees (Pan troglodytes schweinfurthii). Behav. Ecol. Sociobiol. 55, 332-340.

Piccione, G., Giannetto, C., Marafioti, S., Casella, S., Caola, G., 2011. Comparison between circadian motor activity in pony and horse. Rev. chil. hist. nat. 84, 263-268.

Roth, L. S. V., Faresjö, Å., Theodorsson, E., Jensen, P., 2016. Hair cortisol varies with season and lifestyle and relates to human interactions in German shepherd dogs. Sci. Rep. 6, 1-7.

Russell, E., Koren, G., Rieder, M., Van Uum, S., 2012. Hair cortisol as a biological marker of chronic stress: Current status, future directions and unanswered questions. Psychoneuroendocrinology, 37, 589-601.

Steudte, S., Stadler, T., Dettenborn, L., Klumbies, E., Foley, P., Beesdo-Baum, K., Kirschbaum, C., 2011. Decreased hair cortisol concentrations in generalised anxiety disorder. Psychiatry Res. 186, 310-314.

Sundman, A-S., Van Poucke, E., Svensson Holm, A-C, Faresjö, Å., Theodorsson, E., Jensen, P., Roth, LSV. 2019. Long-term stress levels are synchronized in dogs and their owners. Sci Reports, 9:7391.

Thorell, G., Hedenborg, S., 2015. Riding Instructors, Gender, Militarism, and Stable Culture in Sweden: Continuity and Change in the Twentieth Century. Int. J. Hist. Sport. 32, 650-666.

Viksten, S.M., Visser, E.K., Nyman, S., Blokhuis, H., 2017. Developing a horse welfare assessment protocol. Anim. Welf. 26, 59-65.

Visser, E.K., Van Wijk-Jansen, E.E.C., 2012. Diversity in horse enthusiasts with respect to horse welfare: An explorative study J. Vet. Behav. 7, 295-304.

Yarnell, K., Hall, C., Royle, C., Walker, S.L., 2015. Domesticated horses differ in their behavioural and physiological responses to isolated and group housing. Physiol. Behav. $143,51-57$. 


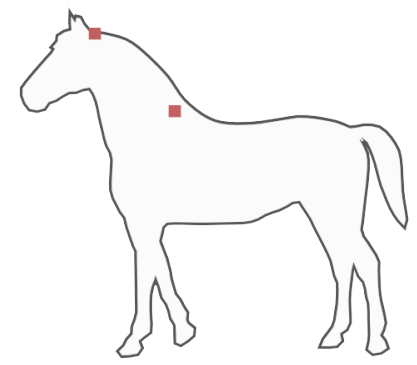

Figure 1. The body locations of the two hair samples obtained from the horses.

507

508

509
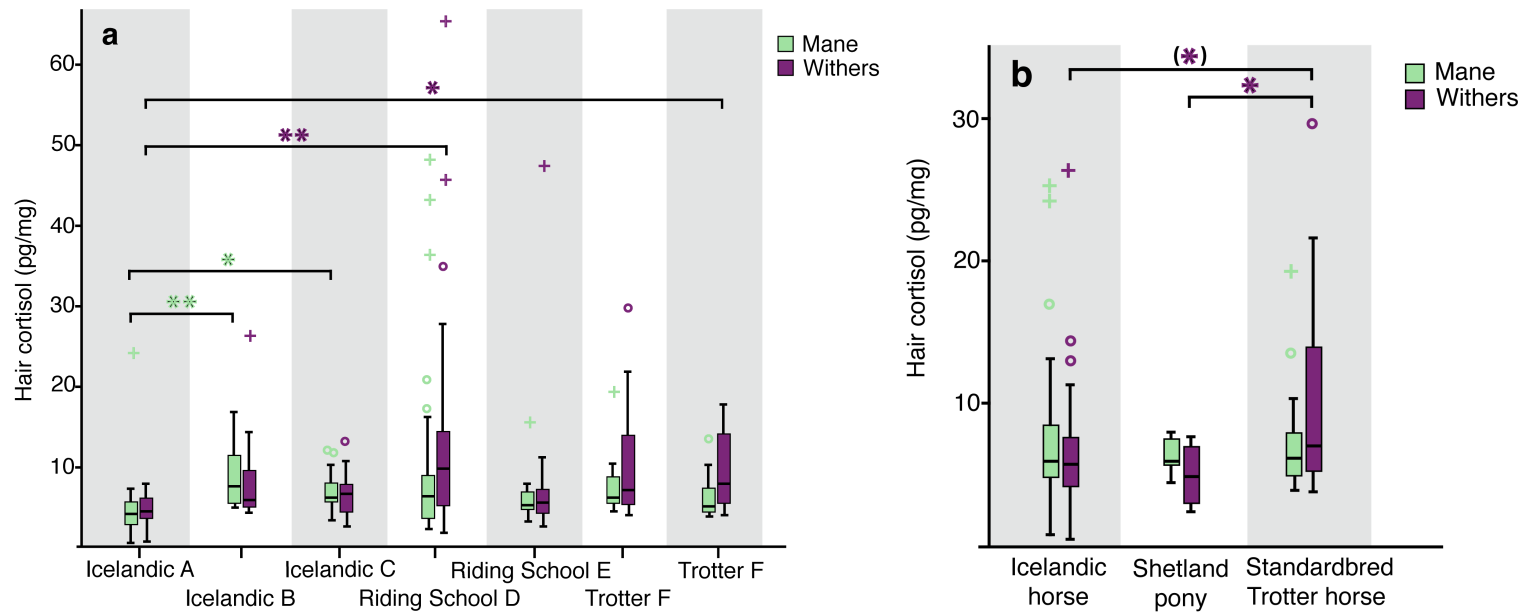

Figure 2. Hair cortisol concentrations $(\mathrm{pg} / \mathrm{mg}$ ) in the different stables and breeds. Boxplots with interquartile ranges. The bars in the boxes indicate the median for each stable $(A)$ and breed $(B)$ for both mane and withers hair. Circles indicate outlier and plusses specify extreme outliers (three times the interquartile range from a quartile). ${ }^{*} P<0.01, * P<0.05$ and $\left({ }^{*}\right) \mathrm{P}<0.06$ for both mane HCC and withers HCC. 

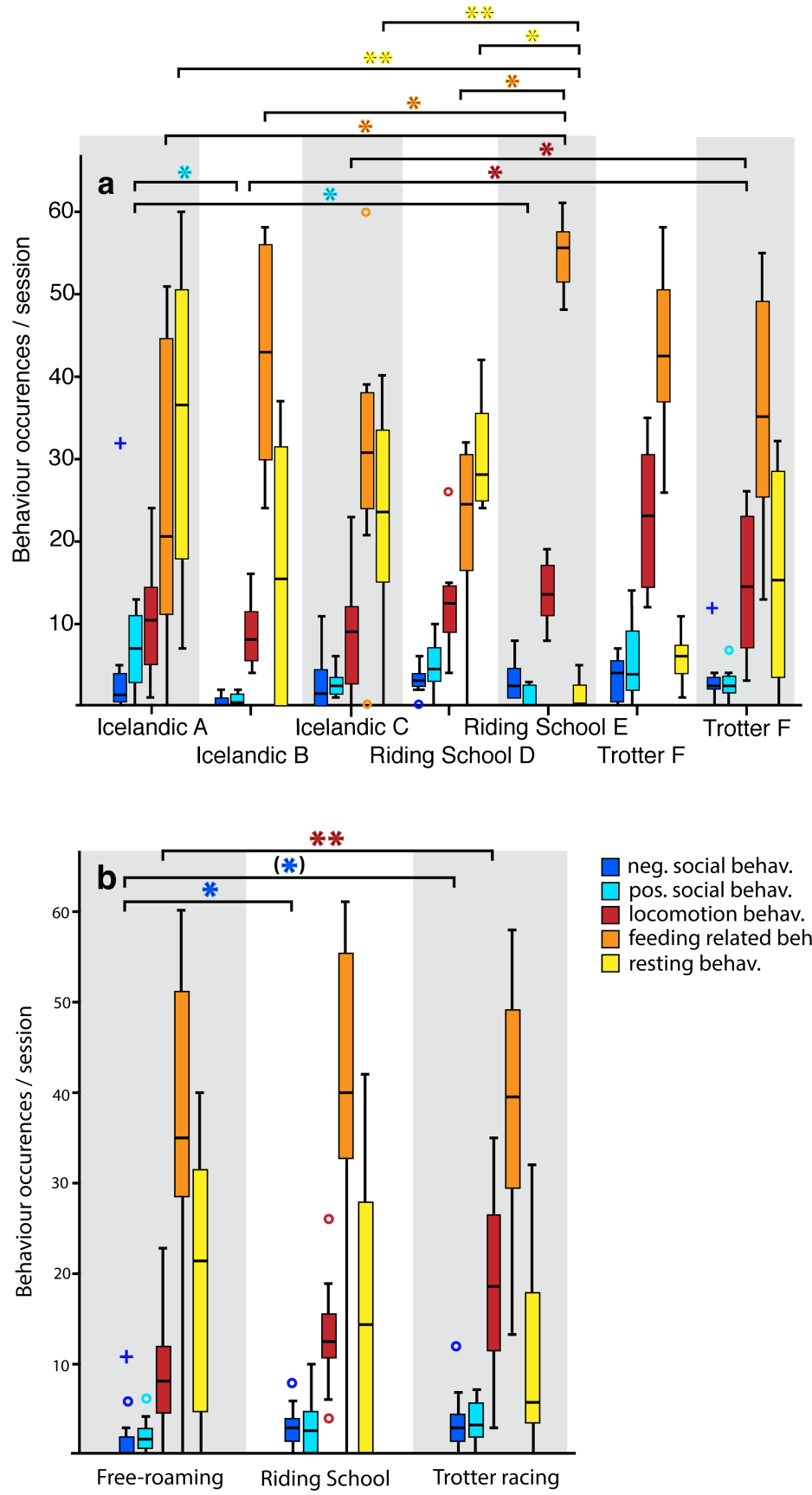

$$
\begin{aligned}
& \square \text { neg. social behav. } \\
& \square \text { pos. social behav. } \\
& \square \text { locomotion behav. } \\
& \square \text { feeding related behav. } \\
& \square \text { resting behav. }
\end{aligned}
$$

Free-roaming Riding School Trotter racing

Figure 3. Results from behavioural observations. Boxplots with interquartile ranges. The bars in the boxes indicate the median of behavioural occurrences for each behaviour category for the different stables (A) and management regimes (B). Circles indicate outliers and plusses specify extreme outliers (three times the interquartile range from a quartile). ${ }^{*} P<<0.01,{ }^{*} P<0.05$ and $\left({ }^{*}\right) P<0.06$ for all behaviours. 
531 Table 1. Stables and mean ages $( \pm \mathrm{SE}$ ) of horses included in this study.

\begin{tabular}{lllr}
\hline Stable & Management regime & N & Mean age (years) \\
\hline Icelandic A & - & 16 & $15.9 \pm 1.4$ \\
Icelandic B & Free-roaming & 14 & $14.8 \pm 1.6$ \\
Icelandic C & Free-roaming & 25 & $12.9 \pm 1.3$ \\
Riding school D & Riding school & 46 & $12.9 \pm 0.7$ \\
Riding school E & Riding school & 23 & $9.9 \pm 0.8$ \\
Trotter F & Trotter racing & 16 & $6.3 \pm 1.4$ \\
Trotter G & Trotter racing & 13 & $5.2 \pm 0.5$ \\
\hline
\end{tabular}




\begin{tabular}{|c|c|c|}
\hline Behaviour & Definition & Sampling method \\
\hline \multicolumn{3}{|c|}{ Negative Social behaviour } \\
\hline Biting & Reaching for conspecific with an open mouth while flattening ears & Continuous \\
\hline Kicking & $\begin{array}{l}\text { One or two legs extended in the air towards conspecific while } \\
\text { flattening ears }\end{array}$ & Continuous \\
\hline Pushing & Moving a conspecific by applying physical pressure to its body & Continuous \\
\hline Head threat & Extending neck towards conspecific while flattening ears & Continuous \\
\hline Stomping & Stamping one or two feet against the ground near a conspecific & Continuous \\
\hline \multicolumn{3}{|c|}{ Positive Social behaviour } \\
\hline Mutual sniffing & Nostrils of two conspecifics are in immediate proximity & Continuous \\
\hline Flehmen response & Extending neck and rolling back upper lip, exposing teeth & Continuous \\
\hline Play behaviour & $\begin{array}{l}\text { Biting, kicking as previously described but with ears mobile, in forward } \\
\text { or axial position. Also includes pursuit of a conspecific }\end{array}$ & Continuous \\
\hline Mutual grooming & $\begin{array}{l}\text { Two horses simultaneously nipping or rubbing each other's neck, } \\
\text { withers and/or back }\end{array}$ & Continuous \\
\hline One-way grooming & $\begin{array}{l}\text { One horse nipping or rubbing a conspecific's neck, withers and/or } \\
\text { back. Includes horse nipping or rubbing its own body }\end{array}$ & Continuous \\
\hline \multicolumn{3}{|c|}{ 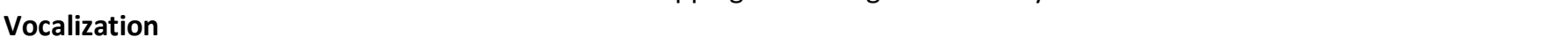 } \\
\hline Neighing & Long and loud sound emitted by a horse with mouth slightly open & Continuous \\
\hline Snorting & Expulsion of air through the nostrils creating a repetitive sound & Continuous \\
\hline \multicolumn{3}{|c|}{ Stereotypic behaviour } \\
\hline Crib-biting & $\begin{array}{l}\text { Putting pressure on stationery structure with teeth then inhaling } \\
\text { deeply }\end{array}$ & Continuous \\
\hline Weaving & Shifting body weight from side to side repeatedly & $1 / 0(30 s)$ \\
\hline Pacing & Walk/trot or canter back and forth repeatedly in a given location & $1 / 0(30 \mathrm{~s})$ \\
\hline \multicolumn{3}{|l|}{ Locomotion } \\
\hline Walk & Four beat movement where two or three hooves are on the ground & $1 / 0(30 s)$ \\
\hline Trot & Two beat movement where two or no hooves are on the ground & $1 / 0(30 s)$ \\
\hline Canter/Gallop & $\begin{array}{l}\text { Three beat movement where two or three hooves are on the } \\
\text { ground/Four beat movement where all hooves are off the ground in } \\
\text { each stride }\end{array}$ & $1 / 0(30 \mathrm{~s})$ \\
\hline \multicolumn{3}{|l|}{ Resting/Still } \\
\hline Lying down & $\begin{array}{l}\text { Horse is on the ground with four legs and body in contact with the } \\
\text { ground }\end{array}$ & $1 / 0(30 s)$ \\
\hline Resting & $\begin{array}{l}\text { Body supported by at least } 3 \text { legs, ears rotating laterally, eyelids } \\
\text { drooping, Neck may drop below horizontal }\end{array}$ & $1 / 0(30 s)$ \\
\hline Alert & $\begin{array}{l}\text { Body supported by at least } 3 \text { legs, head held higher than neck, eyes } \\
\text { wide open }\end{array}$ & $1 / 0(30 s)$ \\
\hline Withdrawn & $\begin{array}{l}\text { Body supported by at least } 3 \text { legs, stretched neck, similar neck and } \\
\text { back height. Eyes wide open and no ear or head movements }\end{array}$ & $1 / 0(30 s)$ \\
\hline \multicolumn{3}{|c|}{ Feeding-related behaviour } \\
\hline Grazing/Browsing & $\begin{array}{l}\text { Teeth and lips are in immediate proximity with grass/leaves. Steps } \\
\text { may be taken. }\end{array}$ & $1 / 0(30 \mathrm{~s})$ \\
\hline Eating roughage & Teeth and lips are in immediate proximity with roughage & $1 / 0(30 s)$ \\
\hline Drinking & Lips are in immediate proximity with water & $1 / 0(30 s)$ \\
\hline
\end{tabular}

